Casos Clínicos

Arch. Esp. Urol., 60, 5 (586-588), 2007

\section{INFECCIÓN URINARIA RECURRENTE EN UN CASO DE AGENESIA DEL SACRO Y DISRAFIA VERTEBROLUMBAR. CONDUCTA DIAGNÓSTICOTERAPÉUTICA Y EVOLUCIÓN CLÍNICA.}

Rodolfo Orozco Fariñas, José Ignacio Iglesias Prieto, Jorge Massarrah Halabi, José Mำ Mancebo Gomez y Enrique Perez-Castro Ellendt.

Unidad de Urología. Clínica La Luz. Madrid. España.

Resumen.- OBJETIVO: Presentación y análisis de un caso de agenesia del sacro con bifidez vertebrolumbar distal y reseñar los elementos mas relevantes encontrados en la revisión bibliográfica efectuada.

MÉTODO/RESULTADOS: Se describen las características clínicas de una paciente adulta con agenesia sacra, su manejo urológico diagnósticoterapéutico y evolución durante cuatro años de seguimiento, así mismo, se realiza búsqueda informática de artículos publicados en los últimos 16 años para su revisión, exponiéndose brevemente los elementos mas destacables.
CONCLUSIONES: La agenesia del sacro, como expresión locoregional del "Síndrome de regresión caudal" produce, casi invariablemente, afectación funcional vesical e infección urinaria recurrente, no obstante, como en nuestra paciente, la evolución puede ser benigna, lo que no exime la necesidad ineludible de un correcto diagnóstico morfofuncional inicial, el control periódico y su tratamiento consecuente.

Palabras clave: Agenesia del sacro. Síndrome de regresión caudal. Disgenesia espinal segmentaria.

Summary.- OBIECTIVE: To present and analyze one case of sacral agenesis with distal lumbar vertebral dysraphism, and to highlight the most relevant elements found in a bibliographic search.

METHODS: We describe the clinical characteristics of an adult patient with sacral agenesis, the diagnostic-therapeutic urological management and her outcome over four years of follow-up. A bibliographic search was also performed including review of all articles published over the last 16 years; we briefly include the most relevant elements.

CONCLUSIONS: Sacral agenesis, as a local regional expression of "caudal regression syndrome" almost invariably produces functional involvement of the bladder and recurrent urinary tract infection, although the evolution may be benign like present case. This does not exempt from the unavoidable need of proper initial morphological-functional diagnosis, periodic follow-up and treatment.

Keywords: Sacral agenesis. Caudal regression syndrome. Segmentary spinal dysgenesis.

\title{
INTRODUCCIÓN
}

La agenesia del sacro es una entidad infrecuente $(1,2)$, el defecto primario es la alteración en el desarrollo embrionario del mesodermo del eje medioposterior antes de la $4^{\underline{a}}$ semana gestacional (3); conceptualmente la terminología y clasificación utilizadas para la misma ha sido variada por formar parte de un grupo heterogéneo de anomalías $(2,4,5)$; actualmente se incluye dentro de las disostosis que abarcan, desde las disgenesias espinales segmentarias caudales mas simples, p.ej. agenesia coccígea asintomática y agenesia parcial sacra, hasta los casos mas complejos,

graves o letales como los llamados "niños budas", la

Rodolfo Orozco Fariñas

Clínica "La Luz".

General Rodrigo, 8

28003 Madrid. (España).

drorozco9@hotmail.com

Trabajo recibido: 9 de octubre 2006. 
agenesia toracolumbosacra y la "sirenomielia", enmarcados todos dentro del término "Síndrome de regresión caudal" propuesto por Duhamel; por ende, la agenesia sacra puede aparecer como única alteración locorregional, formar parte de anomalías generalizadas (p.ej. Acondroplasia), o de síndromes específicos como el de Currarino (tumor presacro y anomalía anorectal) o el de Holt Oram (anomalías cardíacas y musculoesqueléticas de miembros superiores), (4-10). De acuerdo con lo anterior su etiopatogenia es variable, compleja y casi desconocida; se han comprobado dos factores predominantes que son: las alteraciones genéticas (hereditarias 0 , mas frecuentemente, esporádicas), y materno teratogénicos (diebetes mellitus, sarampión gestacional); tambien se discuten factores ambientales e isquémicos locales durante la embriogenia $(2,4,6,10-16)$. Dependiendo de la complejidad del caso, el tratamiento puede llegar a ser difícil, multidisciplinario y prolongado $(10,17)$.

\section{CASO CLÍNICO}

Mujer, de 40 años de edad, que es atendida en nuestra consulta en marzo de 2002 por infecciones recurrentes del tracto urinario con cuadro pielonefrítico en 2001 y hallazgo de vejiga con "pólipos" en ecografía ginecológica reciente.

Al interrogatorio no se constatan antecedentes patológicos personales destacables, excepto la referencia de anomalía lumbosacra (espina bífida y agenesia sacra) sin acción diagnósticoterapéutica específica posterior. Dentro de los antecedentes familiares resulta unicamente significativo el de madre portadora de riñón trasplantado por "riñones poliquísticos" sin otras enfermedades asociadas.

Se procede al estudio de la paciente con los siguientes resultados:

- Exploración física general: Cifoescoliosis importante compensada.

- Exploración urológica: inspección lumboabdominal normal. Riñones no palpables, puñopercusión negativa. Puntos pielorenoureterales no dolorosos. Hipogastrio normal a la palpación. Meato uretral normal. Genitales externos sin alteraciones y ausencia de prolapsos genitourinarios. Test de esfuerzo (Mashall-Bonney) negativo.

- Sistemático de sangre y hemoquímica: normales (creatinina en $0.8 \mathrm{dg} / \mathrm{ml}$ y glicemia en $88 \mathrm{mmol} / \mathrm{l}$ ).

- Sistemático de orina: densidad en 1020, PH de 5, leucocituria( 15-20 l/c), 2-3 htíes/c, y bacteriuria marcada, en el sedimento.

- Urocultivo: Echerichia Coli,(mas de 100000 ufc/ ml).

- Ecografía abdominal: riñones y demás órganos in- trabdominales normales, excepto vejiga de aspecto multidiverticular.

- Urografía intravenosa: espacio "vacío" a partir de vértebras L4-L5 con disrafia o bifidez de estas y ausencia de sacro. Morfología y función renal bilateralmente normal, sin anomalías ureterales. Vejiga piriforme o "en abeto", multidiverticular. Ausencia de reflujo vesicoureteral. Residuo postmiccional radiológicamente significativo.

- Estudio urodinámico: no evidencias de incontinencia urinaria por "contracciones no inhibidas" ni "por esfuerzo". Vejiga hiporrefléxica con adaptabilidad moderadamente disminuída, sin afectación de la capacidad vesical ni residuo posmiccional.

- Ecografía vesical postmiccional comprobatoria en consulta: Ausencia de residuo vesical.

Manejo urológico ulterior: tratamiento de la bacteriuria con norfloxacino. Control mensual con análisis general de orina. Urocultivo trimestral. Control urológico general anual. Actitud consecuente con resultados.

Evolución: molestias miccionales ocasionales (una o dos veces al año) que desaparecen con monodosis antibiótica. Estudios de control negativos.

\section{DISCUSIÓN}

En nuestra paciente se comprobó la existencia de una agenesia sacra con disrafia de lumbares $4^{a}$ y $5^{\mathrm{a}}$ como manifestaciones locales, sin anomalías asociadas ni antecedentes familiares que indujeran a un estudio genético por posible enfermedad hereditaria, tampoco se detectó antecedentes maternos de diabetes ni de enfermedad gestacional o factores ambientales con potencial teratogénico; el antecedente de una poliquistosis renal materna, en nuestra opinión, carece de importancia etiopatogénica ya que las alteraciones genéticas de ambas patologías son diferentes; en la literatura médica solo encontramos reportes de anomalía renal ectópica y de fusión asociadas a la agenesia sacra $(14,18-21)$. La agenesia sacra sin otras anomalías se ha observado en el $40-72 \%$ de los casos y presenta mejor pronóstico $(1,5)$. La escoliosis, presente en este caso, forma parte de la historia natural en este tipo de agenesia sacra (22) y el disrafismo ha sido comprobado en mas de la mitad de los casos $(5,17)$.

El diagnóstico básico de esta entidad se establece mediante: cuadro sintomático, exploración física, ultrasonido, Rx simple, resonancia magnética nuclear, y pruebas urodinámicas $(1,10,17,23)$, la electromiografía puede ser útil en determinandos casos (24). Creemos que para el urólogo la ecografía del tracto urinario, la valoración analítica funcional renal, el uroanalisis con cultivo y las pruebas urodinámicas, son ineludibles. 
En el caso que discutimos es de señalar que el aspecto urográfico de la vejiga hacía presuponer la presencia de una vejiga hiperreflexica, situación que puede presentarse en el $35 \%$ de los pacientes (1), no obstante, esto no se constató urodinámicamente en nuestra enfer$\mathrm{ma}$, tampoco el residuo "radiológico". Es conocido que prácticamente el $100 \%$ de los pacientes con agenesia sacra total presentan vejiga neurogénica $(1,7,17,25)$, incluso en las anomalías parciales o leves (S4-S5), se observan disfunciones vesicouretrales severas $(26)$; los casos mas graves pueden requerir tratamiento quirúrgicos como la cistoplastia, o la miotomía vesical complementada con catererismo intermitente $(27,28)$; al respecto, es destacable el hecho de que en nuestra paciente no fueron necesarias medidas específicas. La asociación de disfunción vesical e infección urinaria es muy frecuente y puede ocasionar deterioro de la función renal, sobre todo si existe reflujo vesicouretral, por lo que el control periódico indefinido es imprescindible $(1,17)$; también en este aspecto el comportamiento evolutivo de la paciente fué benigno; varios autores han verificado que el $20-25 \%$ de estos pacientes, con tracto urinario superior inicialmente indemne, presentan deterioro ulterior del mismo (1). La incontinencia urinaria, también frecuente en pacientes portadores de agenesia del sacro, tampoco fue corroborada en nuestra paciente, por lo que el manejo urológico solo se encaminó a la erradicación de la bacteriuria y la vigilancia activa.

\section{CONCLUSIONES}

Para tomar la adecuada decisión terapéutica urológica es mandatorio un correcto estudio morfofuncional completo del paciente, la imagenología puede ser engañosa. La agenesia sacra completa puede tener una evolución benigna pero los controles periódicos son imprescindibles.

\section{BIBLIOGRAFÍA y LECTURAS RECOMENDADAS (*lectura de interés $y^{* *}$ lectura fundamental)}

**1. ABASCAL JUNQUERA, J. M. y cols.: "Evolución urológica de pacientes con agenesia de sacro: 20 años de seguimiento". Arch. Esp. Urol., 59: 595 , 2006.

**2. DUHAMEL, B. y cols.: "The caudal regresión syndrome". J. Ultrasound Med., 21: 915, 2002.

*3. ASLAN, H. y cols.: "Prenatal diagnosis of Caudal Regression Syndrome: a case report". BMC Pregnancy Childbirth, 1: 8, 2001.

*4. GREGOIRE, A.; ZERDANI, S.: "Lumbo-sacral agenesis. Clinical analysis and treatment relating to 4 observations". Pediatr. Med. Chir., 23: 89, 2001.

5. GUILLE J T et als. Lumbosacral agenesis: a new classification correlating spinal deformity and ambulatory potential. J. Bone Joint Surg Am, 84.A:32, 2002.

6. KOCHLING, J.; KARBASIYAN, M.; REIS, A.: "Spectrum of mutations and genotype-phenotype analysis in Currarino Syndrome”. Eur. J. Hum. Genet., 9: 599, 2001
*7. TORTORI-DONATI, P. y cols.: "Segmental spinal dysgenesis: neuroradiologic findings with clinical and embryologic correlation". AJNR Am. J. Neuroradiol., 20: 445, 1999.

8. GONDRES ARGOTE, R. y cols.: "Síndrome de Holt y Oram. Presentación de un caso". Rev. Cub. Ortop. Traumatol., 14: 56, 2000.

*9. GARCÍA BARRECHEGUREN, E.; ALFONSO OLMOS, M.; BERIGUISTAIN GURPIDE.: "Defecto vertebral múltiple con otras anomalías acompañantes". Anales Esp. Pediat., 54: 409, 2001.

*10. SINGH S.K.; SINGH R.D.; SHARMA, A.: "Caudal regression Syndrome -Case report and review of literature". Pediatr. Surg. Int., 21: 578, 2005.

11. WANG, J. y cols.: "Sacral dysgenesis associated with terminal deletion of chromosome 7q: a report of two families". Eur. J. Pediatr., 158: 902, 1999.

12. PAPAPETROU, C. y cols.: "A genetic study of the human $\mathrm{T}$ gene and its exclusion as a major candidate gene for sacral agenesis with anorectal atresia". J. Med. Genet., 36: 208, 1999.

13. FUNAYAMA, C. y cols.: "Recurrent meningitis in a case of congenital anterior sacral and coccygeal vertebrae". Arq. Neuropsiquiatr., 53: 799, 1995.

14. PODESTÁ, M.R.; SILVIA, W.; JUAN, B.A.: “¿Cuál es su diagnóstico?”. Rev. Hosp. de niños de Buenos Aires, XXXIX: 212, 1997.

*15. VALENZANO, M. y cols.: "Sirenomelia. Pathological features, antenatal ultrasonographic clues, and a review of current embryogenic theories". Hum. Reprod. Update., 5: 82, 1999.

16. SOOD, R. y cols.: "Cloning and characterization of an inversion breakpoint at $6 \mathrm{q} 23.3$ suggest a role for map 7 in sacral dysgenesis". Cytogenet. genoma Res., 106: 61, 2004.

**17. GOTOH, T. y cols.: "Diagnosis and manegement of sacral agenesis". Eur. Urol., 20: 287, 1991.

18. LIMB, J.; BELLMAN, G.C.: "Percutaneous management of stones in a patient with sacral agenesis". Urology, 56: 1056, 2000.

19. GOFRIT, O.N.; PODE, D.; LANDAU, E.H.: "Vesicocolonic fistula four years after augmentation colocystoplasty". Urol. Int., 62: 117, 1999.

20. MARTUCCIELLO, G. y cols.: "Currarino syndrome. Proposal of a diagnostic and Therapeutic protocol". Review J. Pediatr. Surg., 39: 1305, 2004.

21. BRINKMANN, O.A.; KUWERTZ-BROKING, E.; HERTLE, L.: "Complex malformation: cake kidney with concomitant sacral agenesis". Aktuelle Urol., 33: 64, 2002

*22. VAN BUSKIRK, C.S.; RITTERBUSCH, J.F.: "Natural history of distal spinal agenesis". J. Pediatr. Orthop. B., 6: 146, 1997

23. LEE, M.C.; WANG, P.J.; CHEN, R.L.: "Lumbosacral agenesis in a premature infant of a diabetic mother". Acta Paediatr. Taiwan., 44: 375, 2003.

*24. PODNAR, S.: "Wich patients need referral for anal sphincter electromyography?". Muscle Nerve, 33: 278, 2006.

**25. WILMSHURST, J.M.; KELLY, R.; BORZYSKOUSKI, M.: "Presentation and outcome of sacral agenesis: 20 years' experience". Urology, 56: 1056, 2000.

**25. BOEMERS, T.M. y cols.: "Urology problems in anorectal malformations. Part 1: urodynamic findings and significance of sacral anomalies". J. Pediatr. Surg., 31: 407, 1996.

26. HEDICAN, S.P.; SCHULAM, P.G.; DOCIMO, S.G.: "Laparoscopic assisted reconstructive surgery". J. Urol., 161: 267, 1999. 
27. RAWASHDEH, Y.F.; JORGENSEN, T.M.; OLSEN, L.H. y cols.: "The outcome of detrusor myotomy in children with neurogenic bladder dysfunction". J. Urol., 171: 2654, 2004.

Casos Clínicos

Arch. Esp. Urol., 60, 5 (589-593), 2007

\section{ANEURISMA CIRSOIDE RENAL: UNA RARA CAUSA DE HEMATURIA SEVERA.}

José Gregorio Pereira Arias, Vicente Ullate Jaime, Esther Pereda Martínez', José María Gutiérrez Díez, Ricardo Ateca Díaz-Obregón, Maria Mar Ramírez Rodríguez’ y José Ramón Berreteaga Gallastegui.

Servicio de Urología. Sección de Anatomía Patológica'. Hospital de San Eloy. Baracaldo. Vizcaya. España.

Resumen.- OBJETIVO: Las fístulas arteriovenosas congénitas renales (aneurisma cirsoide) son una realidad clínica excepcional. Aunque con frecuencia son asintomáticas, su presentación como hematuria grave suscita un excelente ejercicio diagnóstico, y a menudo una actuación terapéutica inmediata.

MÉTODOS Y RESULTADOS: Presentamos una paciente de 75 años de edad con un cuadro de hematuria severa anemizante, hipertensión arterial e insuficiencia cardiaca con- gestiva. El diagnóstico de imagen revelo una ureterohidronefrosis renal derecha con bloqueo vesical por coágulos. El estudio endoscópico (cistoscopia y ureterorenoscopia) alertó sobre el origen renal derecho de la hemorragia, precisando finalmente una nefrectomía resolutiva. El diagnóstico histológico objetivó la presencia de una formación redondeada de múltiples canales vasculares arteriales y venosos en la submucosa pielocalicial con erosión focal epitelial, compatible con fístula arteriovenosa congénita renal (aneurisma cirsoide). Revisamos aspectos diagnósticos y terapéuticos en la literatura.

CONCLUSIONES: Las fístulas arteriovenosas congénitas renales representan un dilema diagnóstico. Pueden presentarse de forma asintomática o bien condicionar clínica derivada del shunt y alto gasto cardiaco (cardiopatía hipertensiva e insuficiencia cardiaca congestiva) o por erosión e infiltración hemorrágica aguda de la vía urinaria (hematuria renal severa). El tratamiento debe ser conservador mediante la embolización o esclerosis supraselectiva. No obstante, ante fístulas voluminosas, repermeabilización postembolización o en situaciones de inestabilidad hemodinámica, la nefrectomía es una excelente alternativa.

Palabras clave: Fístula arteriovenosa rena. Aneurisma cirsoide renal.

Summary.- OBJECTIVE: Congenital arteriovenous fistulas are an exceptional clinical feature. Although they are frequently asymptomatic, their presentation as severe hematuria pose an excellent diagnostic exercise and often immediate therapeutic action.

METHODS/RESULTS: We report the case of a 75-year-old female patient presenting with severe hematuria producing anaemia, high blood pressure and congestive heart failure. Image tests revealed right ureteral-hydronephrosis with bladder blockage by blood clots. The endoscopic study (cystoscopy and ureterorenoscopy) alerted about the origin of the hematuria from the right kidney, finally requiring nephrectomy as definitive treatment. Pathology revealed the presence of a round formation with multiple vascular channels, arterial and venous, in the pyelocalicial submucosa, with focal epithelial erosion, compatible with congenital arteriovenous fistula. We review the diagnostic and therapeutic features in the literature.

CONCLUSIONS: Renal congenital arteriovenous fistulas represent a diagnostic dilemma. They may present asymptomatic or condition clinical features derived from the shunt and high cardiac output (hypertensive cardiopathy and congestive heart failure) or from the erosion and acute hemorrhage into the urinary tract (severe renal hematuria). Treatment should be conservative with embolization or supraselective 